\title{
ON MATROID THEOREMS OF EDMONDS AND RADO
}

\author{
D. J. A. WELSH
}

\section{Introduction}

In this note I show how very general and powerful results about the union and intersection of matroids due to J. Edmonds [19] may be deduced from a matroid generalisation of Hall's theorem by R. Rado [13].

Throughout, $S, T$, will denote finite sets, $|X|$ will denote the cardinality of the set $X$ and $\left\{x_{i}: i \in I\right\}$ denotes the set whose distinct elements are the elements $x_{i}$.

A matroid $(S, \mathbf{M})$ is a finite set $S$ together with a family $\mathbf{M}$ of subsets of $S$, called independent sets, which satisfies the following axioms

(1) $\varnothing \in \mathbf{M}$.

(2) If $X$ is independent and $Y \subset X$, then $Y$ is independent.

(3) If $X=\left\{x_{1}, \ldots, x_{m}\right\}$ and $Y=\left\{y_{1}, \ldots, y_{m+1}\right\}$ are members of $\mathbf{M}$ then there exists an element $y_{i}$ of $Y-X$ such that $\left\{x_{1}, \ldots, x_{m}, y_{i}\right\} \in \mathbf{M}$.

It is easy to verify that these axioms are equivalent to many other sets of axioms given by Whitney [18] or Rado [14]. We often write $\mathbf{M}$ for the matroid $(S, \mathbf{M})$ and call $\mathbf{M}$ a matroid on $S$. The rank of a subset $X$ of $S$ is the cardinality of a maximal independent subset of $X$ and is denoted by $r(X)$. The rank of the matroid is $r(S)$ and we often write this as $r(\mathbf{M})$. A base of $(S, \mathbf{M})$ is a maximal independent subset of $S$, and a well-known property of matroids is that if $I$ is any independent set and $B$ is any base, then there exists a subset $Y$ of $B$ such that $I \cup Y$ is also a base.

Associated with any matroid $(S, \mathbf{M})$ is a dual matroid $\left(S, \mathbf{M}^{*}\right)$ which is defined to have as its bases all sets of the form $S-B$, where $B$ is a base of $\mathbf{M}$. Clearly the dual $\mathbf{M}^{*}$ is unique and it is not difficult to show that the rank functions $r$ and $r^{*}$ of a matroid and its dual are connected by

$$
r^{*}(S-A)=|S|-|A|-r(S)+r(A)
$$

for all subsets $A$ of $S$.

We also point out that if $A \subset S$, then any matroid $\mathbf{M}$ on $S$ induces a matroid $\mathbf{M} \times A$ on $A$ in the natural way. $\mathbf{M} \times A$ consists of those subsets of $A$ which are members of $\mathbf{M}$. It is called the reduction of $\mathbf{M}$ to $A$ and clearly its rank function $r_{A}$ is related to the rank function $r$ of $\mathbf{M}$ by

$$
r_{A}(B)=r(B)
$$

for all subsets $B$ of $A$.

Now if $\mathbf{M}_{1}, \mathbf{M}_{2}, \ldots, \mathbf{M}_{k}$ are matroids on $S$ let $\mathbf{M}_{1} \vee \mathbf{M}_{2} \vee \ldots \vee \mathbf{M}_{k}$ denote the collection of subsets of $S$ of the form $X_{1} \cup X_{2} \cup \ldots \cup X_{k}$ where $X_{i} \in \mathbf{M}_{i},(1 \leqslant i \leqslant k)$.

Received 23 October, 1968. Research supported in part by a grant from the Office of Naval Research.

[J. LoNDON MATH. Soc. (2), 2 (1970), 251-256] 
Edmonds [19] has proved that $\mathbf{M}_{1} \vee \ldots \vee \mathbf{M}_{k}$ is a matroid on $S$. Also if $r_{i}$ denotes the rank function of $\mathbf{M}_{i}$, and $R_{k}$ denotes the rank function of $\mathbf{M}_{1} \vee \ldots \vee \mathbf{M}_{k}$ it is easy to see that for any subset $A$ of $S$,

$$
R_{k}(S) \leqslant r_{1}(A)+r_{2}(A)+\ldots+r_{k}(A)+|S-A|,
$$

for let $B$ be any base of $\mathbf{M}_{1} \vee \ldots \vee \mathbf{M}_{k}$, then for any subset $A$ of $S$

$$
R_{k}(S)=|B|=|B \cap A|+|B \cap(S-A)| \leqslant r_{1}(A)+\ldots+r_{k}(A)+|S-A| .
$$

The union theorem of Edmonds is

THEOREM 1. The rank of $\left(S, \mathbf{M}_{1} \vee \mathbf{M}_{2} \vee \ldots \vee \mathbf{M}_{k}\right)$ is given by

$$
R_{k}(S)=\min _{A \subset S}\left[r_{1}(A)+\ldots+r_{k}(A)+|S-A|\right] .
$$

COROLlary. The rank function $R_{k}$ of $\left(S, \mathbf{M}_{1} \vee \ldots \vee \mathbf{M}_{k}\right)$ is given by

$$
R_{k}(A)=\min _{B \subset A}\left[r_{1}(B)+\ldots+r_{k}(B)+|A-B|\right],
$$

for all subsets $A$ of $S$.

To see how (7) follows from (6) it is sufficient to notice that for any subset $A$ of $S$,

$$
\left(A,\left(\mathbf{M}_{1} \times A\right) \vee\left(\mathbf{M}_{2} \times A\right) \vee \ldots \vee\left(\mathbf{M}_{k} \times A\right)\right)=\left(A,\left(\mathbf{M}_{1} \vee \mathbf{M}_{2} \vee \ldots \vee \mathbf{M}_{k}\right) \times A\right)
$$

and since the rank of $A$ in the matroid $(S, M)$ is just the rank of the matroid $(A, \mathbf{M} \times A)$, (7) follows.

Let $S, T$ be finite sets and let $\sim$ be an incidence relation between the elements of $S$ and the elements of $T$. If $s \in S$ and $t \in T$ and $s \sim t$ then we say that $s$ and $t$ are incident. For each $s \in S, \tilde{s}=(t \in T ; s \sim t)$. If $X \subset S$, then $\tilde{X}=\bigcup_{s \in X} \tilde{s}$.

For notational convenience we let

$$
S=\{s(i) ; 1 \leqslant i \leqslant m\} \text { and } T=\{t(j) ; 1 \leqslant j \leqslant n\} .
$$

A matching between $S$ and $T$ with respect to the incidence relation $\sim$ is a pair of subsets $(X, Y)$ where $X=\left\{s\left(i_{1}\right), \ldots, s\left(i_{k}\right)\right\}$ and $Y=\left\{t\left(j_{1}\right), \ldots, t\left(j_{k}\right)\right\}$ such that $i_{p} \neq i_{q}(p \neq q)$, and $j_{p} \neq j_{q}(p \neq q)$, and $s(\alpha) \neq s(\beta) \quad(\alpha \neq \beta), t(\alpha) \neq t(\beta) \quad(\alpha \neq \beta)$ and such that for each $\lambda, 1 \leqslant \lambda \leqslant k, s\left(i_{\lambda}\right) \sim t\left(j_{\lambda}\right)$. The common cardinality of $X$ and $Y$ is called the cardinality of the matching $(X, Y)$. The theory of matchings is a much discussed topic in graph theory, see for example Ore [11].

If now $\mathbf{M}$, and $\mathbf{N}$ are matroids on $S$ and $T$ respectively we say that the pair $(X, Y)$ is an independent matching between $(S, \mathbf{M})$ and $(T, \mathbf{N})$ with respect to the incidence relation $\sim$, if $(X, Y)$ is a matching with respect to $\sim$ and $X$ is independent in $(S, M)$ and $Y$ is independent in $(T, N)$.

A " matching" form of Edmonds' intersection theorem as described by Brualdi $[1]$ is

THEOREM 2. If $r_{1}, r_{2}$ denote the rank functions of $(S, \mathbf{M})$ and $(T, \mathrm{~N})$ respectively, then the maximum cardinality of an independent matching between $(S, M)$ and $(T, \mathbf{N})$ with respect to an incidence relation $\sim$ is equal to

$$
\min _{A \subset S}\left[r_{2}(\tilde{A})+r_{1}(S-A)\right] .
$$


Rado's Theorem. Let $\mathbf{A}=\left(A_{i}: 1 \leqslant i \leqslant n\right)$ be any collection of subsets of $S$. Let $\mathbf{M}$ be a matroid on $S$ with rank function $r$. Then if $J \subset(1, \ldots, n)$ and $A(J)$ denotes $\bigcup\left(A_{i}: i \in J\right)$, we have the following result.

THEOREM 3. The collection of subsets $A$ has a transversal which is independent in $\mathbf{M}$ if and only if for all $J \subset(1, \ldots, n)$

$$
r(A(J)) \geqslant|J| \text {. }
$$

A very simple proof of this is given in [17], and also of the following "defect" version due to $H$. Perfect [12].

THEOREM 3'. If $d$ is any non-negative integer $\leqslant n$, then there is a subcollection of $\mathbf{A}$ consisting of all but $d$ of the $A_{i}$ which has a transversal which is independent in $\mathbf{M}$ if and only if for all $J \subset(1, \ldots, n)$

$$
r(A(J)) \geqslant|J|-(n-d) \text {. }
$$

Brualdi [1] shows how Theorem 3 is deduced from Theorem 2.

Deduction of Theorem 1 from Theorem 3. Let $S=\left\{s_{1}, s_{2}, \ldots, s_{n}\right\}$. Let $\mathbf{M}_{i}$ $(1 \leqslant i \leqslant k)$ be matroids on $S$. For $1 \leqslant i \leqslant k$, let $S_{i}$ be disjoint sets with

$$
S_{i}=\left\{s_{1 i}, s_{2 i}, \ldots, s_{n i}\right\} \text {. }
$$

Let $\mathbf{M}_{i}{ }^{\prime}$ be a matroid on $S_{i}$, isomorphic to $\mathbf{M}_{i}$ under the obvious mapping. Then since the $S_{i}$ are disjoint, $\mathrm{V}\left(\mathbf{M}_{i}^{\prime}: 1 \leqslant i \leqslant k\right)$ is a matroid on $S^{\prime}=\bigcup\left(S_{i}: 1 \leqslant i \leqslant k\right)$, with rank function $\rho$ given in terms of the rank functions $r_{i}$ of $\mathbf{M}_{i}$ by

$$
\rho(X)=\sum_{i=1}^{k} r_{i}(X) \text {. }
$$

Let $A_{i}(1 \leqslant i \leqslant n)$ be subsets of $S^{\prime}$ defined by $A_{j}=\left\{s_{i 1}, s_{i 2}, \ldots, s_{i k}\right\}$. Then if $Y \subset \dot{S}$, $Y$ has rank $\geqslant u$ in $V\left(\mathbf{M}_{i}: 1 \leqslant i \leqslant k\right)$ if and only if the collection of subsets $\left(A_{j}: s_{j} \in Y\right)$ have a transversal which has rank $\geqslant u$ in $\mathrm{V}\left(\mathbf{M}_{i}^{\prime}: 1 \leqslant i \leqslant k\right)$. By Theorem $3^{\prime}$, necessary and sufficient conditions for this are that

$$
\rho\left(A_{j_{1}} \cup \ldots, \cup A_{j_{m}}\right) \geqslant m-(|Y|-u)
$$

for any $\left(j_{1}, \ldots, j_{m}\right)$ such that $\left(s_{j_{1}}, \ldots, s_{j_{m}}\right) \subset Y$. But for all $p,(1 \leqslant p \leqslant k)$,

$$
r_{p}\left(A_{j_{1}} \cup A_{j_{2}} \cup \ldots \cup A_{j_{m}}\right)=r_{p}\left(s_{j_{1}}, \ldots, s_{j_{m}}\right) \text {. }
$$

Hence from (8), (9) reduces to the condition that for all $X \subset Y$,

$$
\sum_{i=1}^{k} r_{i}(X) \geqslant|X|-|Y|+u
$$

which completes the proof of Theorem 1 .

Proof of Theorem 2 from Theorem 1 . Let $(S, \mathbf{M})$ and $(T, \mathbf{N})$ be matroids and let $\sim$ be an incidence relation between $S$ and $T$. Construct the bipartite graph $G$ having vertex sets $S \cup T$ with $S \cap T=\varnothing$, and in which the edges of $G$ join a pair of vertices $s \in S, t \in T$, if and only if $s \sim t$. Let $E$ be the edge set of this graph.

For notational reasons we denote a typical member of $E$ by $e(i, j)$, and this will signify that $e$ is the edge joining $s(i)$ of $S$ to $t(j)$ of $T$. 
We let $\mathbf{M}^{\prime}$ be the null set and those subsets

of $E$ for which

$$
\left\{e\left(i_{1}, j_{1}\right), \ldots, e\left(i_{k}, j_{k}\right)\right\}
$$

(i) $s\left(i_{1}\right), s\left(i_{2}\right), \ldots, s\left(i_{k}\right)$ are distinct members of $S$,

(ii) The set $\left\{s\left(i_{1}\right), \ldots, s\left(i_{k}\right)\right\}$ is independent in $(S, M)$.

LeMMA 1. $\mathbf{M}^{\prime}$ is a matroid on $E$.

Proof. If $A \in \mathbf{M}^{\prime}$, then any subset of $A$ is a member of $\mathbf{M}^{\prime}$. Now let

$$
\begin{aligned}
W & =\left\{e\left(i_{1}, j_{1}\right), \ldots, e\left(i_{p}, j_{p}\right)\right\} \\
W^{\prime} & =\left\{e\left(i_{1}{ }^{\prime}, j_{1}{ }^{\prime}\right), \ldots, e\left(i_{p+1}^{\prime}, j_{p+1}^{\prime}\right)\right\}
\end{aligned}
$$

be members of $\mathbf{M}^{\prime}$. Since $\mathbf{M}$ is a matroid on $S$, there exists

$$
s\left(i_{k}{ }^{\prime}\right) \in\left\{s\left(i_{1}{ }^{\prime}\right), \ldots, s\left(i_{p+1}^{\prime}\right)\right\}, \quad \text { such that }\left\{s\left(i_{1}\right), \ldots, s\left(i_{p}\right), s\left(i_{k}{ }^{\prime}\right)\right\}
$$

is an independent subset of $\mathbf{M}$ of cardinality $p+1$. Hence $W \cup\left\{e\left(i_{k}{ }^{\prime}, j_{k}{ }^{\prime}\right)\right\}$ is a member of $\mathbf{M}^{\prime}$ of cardinality $p+1$, and thus $\mathbf{M}^{\prime}$ is a matroid on $E$.

We let $\mathbf{N}^{\prime}$ be the matroid induced on $E$ by $\mathbf{N}$ in the analogous way, and now we can state the obvious lemma

LEMma 2. ( $S, \mathbf{M})$ and $(T, N)$ have an independent matching of cardinality $k$ with respect to $\sim$ if and only if the matroids $\left(E, \mathbf{M}^{\prime}\right)$ and $\left(E, \mathbf{N}^{\prime}\right)$ have a common independent set of cardinality $k$.

We now use Theorem 1 and duality in essentially the same way as Edmonds to prove

THEOREM 4. Two matroids $\left(S, \mathbf{M}_{1}\right)$ and $\left(S_{2}, \mathbf{M}_{2}\right)$ with rank functions $r_{1}$ and $r_{2}$ have a common independent set of cardinality $k$ if and only if for all subsets $A \subset S$,

$$
r_{1}(A)+r_{2}(S-A) \geqslant k \text {. }
$$

Proof. If a subset $I$ is independent in $\mathbf{M}$, and $\mathbf{M}_{2}$ then $S-I$ contains a base of $\mathbf{M}_{2}{ }^{*}$, and thus the rank of the matroid $\mathbf{M}_{1} \vee \mathbf{M}_{2}{ }^{*}$ is not less than $|I|+r_{2}{ }^{*}(S)$.

Conversely if $r\left[\mathbf{M}_{1} \vee \mathbf{M}_{2}{ }^{*}\right] \geqslant k+r_{2}{ }^{*}(S)$, then since any base $B^{*}$ of $\mathbf{M}_{2}{ }^{*}$ is independent in $\mathbf{M}_{1} \vee \mathbf{M}_{2}{ }^{*}$, there must exist a subset $I$ of $S-B^{*}$ which is independent in $\mathbf{M}_{1}$ and has cardinality not less than $k$.

Thus $\mathbf{M}_{1}$ and $\mathbf{M}_{2}$ have a common independent set of cardinality $k$ if and only if

$$
r\left(\mathbf{M}_{1} \vee \mathbf{M}_{2}{ }^{*}\right) \geqslant k+r_{2}{ }^{*}(S) \text {. }
$$

Using Theorem 1 this implies that for any $A \subset S$,

$$
r_{1}(A)+r_{2}{ }^{*}(A)+|S-A| \geqslant k+r_{2}{ }^{*}(S) \text {. }
$$

Using (4), this reduces to

$$
r_{1}(A)+r_{2}(S-A) \geqslant k .
$$

Now combining Lemma 2 and Theorem 4 we see that $(S, M)$ and $(T, N)$ have an independent matching of cardinality $k$ with respect to $\sim$ if and only if

$$
r_{1}{ }^{\prime}(A)+r_{2}{ }^{\prime}(E-A) \geqslant k
$$

for all subsets $A$ of $E$, where $r_{1}{ }^{\prime}, r_{2}{ }^{\prime}$ are the rank functions of $\mathbf{M}^{\prime}$ and $\mathbf{N}^{\prime}$ respectively. 
By the definition of $\mathbf{M}^{\prime}$ and $\mathbf{N}^{\prime}$ on $E$, this is clearly equivalent to

$$
\min _{S_{0} \subset S}\left[r_{2}\left(\tilde{S}_{0}\right)+r_{1}\left(S-S_{0}\right)\right] \geqslant k,
$$

and thus Theorem 2 follows.

\section{Conclusion}

By using the theorems here together with suitably chosen matroids one gets easy proofs of many apparently unrelated combinatorial results. For example, taking $\mathbf{M}_{i}=\mathbf{M}$ for all $i$, we see that the necessary and sufficient conditions for a matroid $\mathbf{M}$ to have $k$ disjoint bases is that $V\left(\mathbf{M}_{i}: 1 \leqslant i \leqslant k\right)$ has a basis of cardinality $k r(S)$, which is so if and only if $\forall A \subset S$,

$$
k r(A)+|S-A| \geqslant k r(S) \text {. }
$$

Similarly $\mathbf{M}$ is such that $S$ is the union of as few as $k$ independent sets if and only if $\forall A \subset S$.

$$
k r(A) \geqslant|A| \text {. }
$$

These results were originally proved for matroids by Edmonds [2] and [3]. By applying (11) when $\mathbf{M}$ is the natural matroid induced on a vector space by linear independence we get the theorem of Horn [6]. By applying (10) and (11) to the cycle matroid of a graph $G$ we deduce the necessary and sufficient conditions (a) for a graph to have $k$ edge disjoint spanning forests and (b) for a graph to be the union of $k$ subforests, thus obtaining graph theorems of Tutte [15] and Nash-Williams [9], [10]. By choosing $\mathbf{M}_{i}$ to be the matroid $\mathbf{M}$ truncated at $r_{i}$, we get necessary and sufficient conditions for a matroid to have disjoint independent sets of $g$ prescribed cardinalities $r_{i}$. Applying this to the special case when $\mathbf{M}$ is a transversal matroid we thus get the result of P. J. Higgins [5] who gives conditions for a family $Q$ of sets to have $k$ mutually disjoint partial transversals of prescribed sizes $n_{1}, n_{2}, \ldots, n_{k}$. Many other covering and packing theorems of this nature proved by Edmonds and Fulkerson [4] follow by a similar argument.

\section{Acknowledgment}

I wish to acknowledge some very helpful correspondence with R. A. Brualdi and C. St. J. A. Nash-Williams.

\section{References}

1. R. A. Brualdi, "Admissible mappings between dependence structures" (to be published).

2. J. Edmonds, "Minimum partition of a matroid into independent subsets", J. Res. Nat. Bur. Standards Sect. B69 (1965), 67-72.

3. - "On Lehman's switching game and a theorem of Tutte and Nash-Williams", J. Res. Nat. Bur. Standards Sect. B69 (1965), 73-77.

4. —_ and D. R. Fulkerson, "Transversals and matroid partition ", J. Res. Nat. Bur. Standards Sect. B69 (1965), 147-153.

5. P. J. Higgins, "Disjoint transversals of subsets ", Canad. J. Math., 11 (1959), 280-285.

6. A. Horn, "A characterisation of unions of linearly independent sets", J. London Math. Soc., 30 (1955), 494-496.

7. L. Mirsky and H. Perfect, "Applications of the notion of independence to problems of combinatorial analysis", J. Comb. Theory, 2 (1967), 327-357. 
8. C. St. J. A. Nash-Williams, "An application of matroids to graph theory", Theory of graphs, International Symposium, Rome, July 1966, Dunod (1967), 263-265.

9. _- "Edge disjoint spanning trees of finite graphs", J. London Math. Soc., 36 (1961), 445-450.

10. __ " "Decomposition of finite graphs into forests ", J. London Math. Soc., 39 (1964), 12.

11. O. Ore, "Graphs and matching theorems", Duke Math. J., 22 (1955), 625-639.

12. H. Perfect, "Independence spaces and combinatorial problems", Proc. London Math. Soc., 19 (1969), 17-30.

13. R. Rado, "A theorem on independence relations", Quart. J. Math. Oxford Ser. 13 (1942), 83-89.

14. —_ "Abstract linear dependence ", Colloq. Math., 14 (1966), 257-264.

15. W. T. Tutte, "On the problem of decomposing a graph into $n$ connected factors ", J. London Math. Soc., 36 (1961), 221-230.

16. D. J. A. Welsh, "Applications of a theorem of Rado ", Mathematika, 15 (1968), 199-203.

17. —_, "Generalised versions of Hall's theorem ", J. Comb. Theory (1970) (to appear).

18. H. Whitney, "On the abstract properties of linear dependence", Amer. J. Math., 57 (1935), 509-533.

19. J. Edmonds " Submodular functions, matroids, and certain polyhedra " Lectures, Calgary International Symposium on Combinatorial Structures, June 1969.

Merton College, Oxford,

and

University of Michigan. 